\title{
Perfil profesional del grado en pedagogía: análisis de las percepciones universitarias
}

Susana Tallón-Rosales ${ }^{1}$, María del Pilar Cáceres-Reche², Gerardo Gómez-García² y Carmen Rodríguez-Jiménez ${ }^{2 *}$

(1) Departamento de Pedagogía, Universidad de Jaén, 23009 Jaén, España. (Correo-e: stallon@ujaen.es)

(2) Departamento de Didáctica y Organización Escolar, Universidad de Granada, 18071 Granada, España

(Correo-e: caceres@ugr.es; gomezgarcia@ugr.es; carmenrj@ugr.es)

* Autor a quien debe ser dirigida la correspondencia.

Recibido Jul. 22, 2021; Aceptado Sep. 27, 2021; Versión final Oct. 28, 2021, Publicado Feb. 2022

\section{Resumen}

Este trabajo analiza las percepciones acerca de la titulación en pedagogía, sus salidas profesionales y ámbitos laborales, desde la visión de futuros pedagogos. La pedagogía como profesión plantea controversias al delimitar su ámbito laboral de desarrollo profesional. Dicha titulación ofrece un enfoque formativo con tendencia hacia lo formal y no hacia otros ámbitos como el emprendimiento, autoempleo y/o sector empresarial. Se implementa una metodología descriptiva y cuantitativa, con un diseño no experimental por método de encuesta, empleando como instrumento el cuestionario. Los participantes son 334 alumnos del grado en pedagogía de la Facultad de Ciencias de la Educación de la Universidad de Granada (España). Los resultados muestran que el alumnado percibe la profesión de la pedagogía centrada en tres funciones: orientación escolar, intervención con necesidades especiales diversas y asesoría didáctica. Se concluye que la pedagogía está enmarcada generalmente en la educación formal, como principal ámbito de desempeño profesional.

Palabras clave: grado de pedagogía; competencias; perfil profesional

\section{Professional profile of the pedagogy degree: analysis of university perceptions}

\begin{abstract}
This study aims to assess perceptions about the pedagogy degree, including professional opportunities and job fields, from the point of view of future teachers. Pedagogy as a profession is controversial when it comes to defining the scope of its professional development. The pedagogy degree provides a training approach with a tendency towards traditional-type jobs and not towards non-traditional jobs including entrepreneurship, selfemployment, and business sector jobs. A descriptive and quantitative methodology is applied with a nonexperimental design by means of a survey method that uses a questionnaire as an instrument. The participants were 334 students enrolled in the pedagogy degree of the Faculty of Education Sciences at the University of Granada (Spain) in the 2019-2020 academic year. The results show that students perceive that professional pedagogy is focused on three main functions: school guidance, special needs interventions, and didactic consultancy. It is concluded that pedagogy is generally framed within traditional-type jobs as the main fields for professional career development.
\end{abstract}




\section{INTRODUCCIÓN}

La titulación de Pedagogía, surgió en el año 1983, como Licenciatura en la provincia de Granada, con la finalidad de atender y ofrecer una mayor formación y especialización a los profesionales del ámbito educativo. En un primer momento, ubicada dentro de la Facultad de Filosofía y Letras (Sección Pedagogía), del mismo modo que las secciones de Filosofía y Psicología. Más tarde, con la creación de la Facultad de Ciencias de la Educación, dicha sección de Pedagogía, se integra junto con la antigua Escuela Universitaria de Formación del Profesorado, en un único centro universitario en la provincia de Granada. El concepto de Pedagogía, desde sus inicios hasta la actualidad, como señala Saldarriaga, sigue la misma línea de interpretación que Nassif (1981), más de 30 años después: resalta el concepto de esta disciplina como conjunto de saberes interdisciplinares como base, indicando que "con el ingreso de los saberes experimentales sobre el hombre y sobre la sociedad, el lugar central de la Pedagogía como ciencia y arte de educar" es desplazado y sustituido por un nuevo estatuto, el de la Pedagogía como una disciplina aplicada, instrumental, de las ciencias experimentales, un estatuto más técnico remitido a un cuerpo de especialistas de la Paidometría y las ciencias de la educación (2017, p.120).

Versan evoluciones claves en el Currículum de la titulación de Pedagogía, para la adaptación a la sociedad y sus demandas; ya hace más de una década, en el Informe sobre la Titulación de Pedagogía (Facultad Ciencias de la Educación Granada, 2003) se destacaba, una estructura curricular meramente centrada en vías de desarrollo profesional para el Ámbito Formal, no mencionando o dejando sin especificar, qué labor puede desarrollar en el Ámbito No Formal. En este sentido, se empieza a considerar una vía más para los pedagogos y pedagogas, relacionado con lo no formal, sus instituciones, la empleabilidad e inserción laboral, pero aún denotamos la ausencia de contenido curricular relacionado con el ámbito empresarial y sus funciones. En este orden de ampliación de funciones a otros contextos afines, estos estudios han sufrido diversos cambios legislativos y de planes de estudios para la mejora de la calidad de los mismos. En definitiva, es una titulación que se nutre de otras ciencias tales, como la Psicología, Filosofía, Sociología, Antropología... cuestión que queda patente en su estructura curricular agrupada en módulos (tabla 1):

Apostar por una formación inicial en la Universidad de manera integral y bajo criterios acordes de calidad, resulta de la inclusión más heterogénea de materiales curriculares que abarquen todos los contextos, Laboral, Educativo, No-formal y Empresarial. Asimismo, et ál., (2011), consideran que "se trata de una titulación cada vez más transversal y aplicable a diferentes contextos de actuación, no exclusivos del ámbito puramente escolar" (2011, p.15). Y es ahí, donde los profesionales de la Pedagogía deben aunar esfuerzos para potenciar sus posibilidades laborales a otros dichos contextos y contribuir a desmitificar su única vinculación con el ámbito educativo formal. De este modo, esta investigación, pretende visibilizar la imagen laboral del profesional de la Pedagogía, a través del análisis de las respuestas ofrecidas por el estudiantado universitario participante, en torno a dimensiones tales como, funciones y competencias atribuidas, conocimiento que versan sobre el propio grado y sus salidas profesionales, así como, concepciones y expectativas.

La Pedagogía es la disciplina, el estudio o el conjunto de normas, que se refieren a un hecho o a un proceso o actividad de la educación. El futuro graduado en Pedagogía, dispondrá de un contenido curricular en torno a varios itinerarios para una primera toma de contacto dentro del Grado, y en su caso especializarse en alguno de ellos, a través de formación acorde de posgrado que brinde continuidad al itinerario escogido (Medina, y González, 2019). En esta misma línea, así lo define Valladares (2020, p.99) "Asumir una perspectiva transdisciplinaria de la Pedagogía, permitirá lograr que los pedagogos del siglo XXI, en su desempeño académico y profesional, atraviesen los límites disciplinarios de las asignaturas que cursarán en su formación, y comprender los distintos campos educativos". Todo ello requerirá del alumnado universitario una exigencia en cuanto al desarrollo de nuevos roles y responsabilidades para mejorar o cambiar determinadas competencias o ideas que se tienen sobre la enseñanza universitaria y los estudios que se están cursando

La Pedagogía como profesión, parte de un hándicap de base, y es la dificultad para definir qué es y qué hace por la sociedad. Numerosas profesiones, tales como Medicina, Psicología, Abogacía, Arquitectura...etc., son socialmente conocida su función y su aplicación práctica. Es decir, no se cuestiona qué son y para qué son, y, además, están respaldados jurídicamente por Colegios Profesionales que defienden y velan por sus derechos. Aquellos estudiantes que escogen la titulación de Pedagogía, suelen estar escasamente informados respecto a la propia función de esta profesión y sus salidas profesionales a las que pueden dedicarse. Por tanto, al finalizar su etapa formativa universitaria, se sienten confusos y desubicados en el proceso de búsqueda de empleo, porque desconocen a qué dedicarse y en qué ámbitos pueden insertarse laboralmente. En este sentido, Santos y Álvarez (2017), enfatizan que "los estudiantes se sienten poco motivados para emprender en el terreno laboral y sus expectativas se ven afectadas por la disociación percibida entre titulación y demandas de trabajo, emiten valoraciones negativas de su formación e inserción" (2017, p.122). 
Tabla 1: Curriculum grado Pedagogía. Adaptado de la web Universidad de Granda (2021)

\begin{tabular}{|c|c|}
\hline Primer Semestre & Segundo Semestre \\
\hline $\begin{array}{l}\text { (FB) Psicología Del Desarrollo } \\
\text { (FB) Sociología del sistema educativo y social } \\
\text { (FB) Teoría de la educación } \\
\text { (FB) Fundamentos de la investigación educativa } \\
\text { (FB) Antropología de la Educación }\end{array}$ & $\begin{array}{l}\text { (FB) Didáctica general } \\
\text { (FB) Psicología de la Educación } \\
\text { (FB) Sociología de las prácticas educativas } \\
\text { (FB) Educación para la Igualdad y la Diversidad } \\
\text { (FB) Historia social y cultural de la Educación }\end{array}$ \\
\hline $\begin{array}{l}\text { (FD) Filosofía de la Educación } \\
\text { (FD) Educación comparada e internacional } \\
\text { (FD) Políticas y reformas educativas } \\
\text { (FD) Historia del Sistema Educativo español } \\
\text { (FD) Diseño, desarrollo e innovación del currículum }\end{array}$ & $\begin{array}{l}\text { (FD) Metodología de la investigación educativa } \\
\text { (FD) Dirección, organización y gestión de centros } \\
\text { (FD) Tecnología educativa } \\
\text { (OP) Sociología de los medios de comunicación } \\
\text { (OP) Educación democrática y ciudadanía } \\
\text { (OP) Asesoramiento educativo en centros } \\
\text { (OP) Didáctica en la Educación Especial }\end{array}$ \\
\hline $\begin{array}{l}\text { (FD) Diagnóstico y Orientación educativa } \\
\text { (FD) Pedagogía social } \\
\text { (PE) Empleabilidad, formación e inserción laboral } \\
\text { (OP) Procesos y técnicas de inter. psicoeducativa } \\
\text { (OP) Educación para la Paz y la sostenibilidad } \\
\text { (OP) Diseño, desarrollo y evaluación de TICs } \\
\text { (OP) Educación y asesoramiento familiar }\end{array}$ & $\begin{array}{l}\text { (FD) Formación y desarrollo profesional } \\
\text { (PE) Visitas, seminarios, cursos y jornadas. } \\
\text { (PE) Transición a la vida laboral activa } \\
\text { (OP) Historia de la educación de las mujeres } \\
\text { (OP) La observación en contextos educativos } \\
\text { (OP) Programas de intervención psicoeducativa } \\
\text { (OP) Inclusión-exclusión social, escolar en } \\
\text { jóvenes }\end{array}$ \\
\hline $\begin{array}{l}\text { (FD) Evaluación de programas, centros y profesorado } \\
\text { (OP) Diagnóstico pedagógico } \\
\text { (OP) Formación de formadores } \\
\text { (OP) Teoría, modelos educativos en drogodependencias } \\
\text { (OP) Museos e instituciones de la memoria } \\
\text { (OP) Liderazgo y calidad en instituciones no formales } \\
\text { (OP) Multialfabetismos y comunicación en red } \\
\text { (OP) Orientación escolar y tutoría } \\
\text { (OP) Migraciones y educación } \\
\text { (OP) La dimensión económica de la Educación }\end{array}$ & $\begin{array}{l}\text { (PE) Prácticas externas } \\
\text { (TFG) Trabajo de Fin de Grado }\end{array}$ \\
\hline
\end{tabular}

\section{OTROS ANTECEDENTES}

La gran mayoría de los estudiantes no pueden ofrecer respuestas acordes a cuestiones sencillas tales como, ¿qué hace un Pedagogo? ¿qué es la Pedagogía? (Petrie, 2005). Para este autor, la ambigüedad que despierta esta profesión, se origina al vincular esta titulación únicamente con el ámbito de educación formal (centros educativos). El desempeño profesional de la Pedagogía en un primer momento, parece asimilarse a las tareas de maestro o profesor, sin profundizar en todas aquellas funciones que este profesional puede y debe ejercer en otros ámbitos y contextos. Pero dichas funciones y contextos de trabajo no son tan conocidos, realmente la sociedad no identifica el desempeño profesional de esta titulación o, ¿es la titulación en sí la que no proyecta adecuadamente su perfil laboral a la sociedad? Es por ello, por lo que se plantea este estudio centrado en el análisis del perfil laboral de la Pedagogía, desde las percepciones del estudiantado universitario de esta titulación. Es de suma importancia, realizarla en el contexto apropiado, es decir, en la entidad encargada de formar a las futuras generaciones de pedagogos y pedagogas, la Universidad (Ermenc, et ál., 2015; Robinson, y Hope, 2013).

En este contexto, es necesario conocer los cambios acontecidos en sus estructuras y contenidos curriculares para alcanzar la efectiva adaptación al Espacio Europeo de Educación Superior, suponiendo nuevos modos de hacer, de aprender y nuevas exigencias al alumnado universitario (Collin, et ál., 2013). Por ello, López, et ál., (2019, p.163), enfatizan al respecto "un incremento en la percepción de carga de trabajo de los estudiantes, y la necesidad de trabajar en las aulas, competencias interpersonales, planificación y organización del trabajo en equipo, incluyendo una mayor coordinación docente entre los sistemas de evaluación". Con ello, destacamos que el ámbito universitario tiene sus propias características a tener en cuenta, independientemente de la especificidad e historia académica de cada titulación impartida. 
El propio concepto de Pedagogía con el devenir de los años, ha ampliado su desempeño profesional desde su origen, atendiendo en exclusividad a una función como guía, ayuda y conducción de los niños, hasta la actualidad, donde se enfatizan más otra serie de ámbitos, pero no únicamente el educativo, como pueden ser, el no formal y empresarial, siempre y cuando estén presentes procesos de Enseñanza-Aprendizaje (Miller y Baker, 2007). En esta línea de trabajo, autores como Zhuina (2014) y Tolipov (2019) perfilan una perspectiva más pragmática de las nuevas funciones de la Pedagogía y de su actuación profesional, como asesor en la empresa del siglo XXI, como experto en la orientación y búsqueda de empleo, como formador de formadores y asesor y como profesional formado en tecnologías y su aplicación para generar contextos innovadores.

Es en dichos contextos fuera del ámbito educativo, donde curiosamente esta profesión tiene multitud de funciones y tareas por acometer, Por ello, Zhuina (2014) destaca, la necesidad existente que hay de una mayor presencia de contenidos que versen sobre las salidas profesionales, sobre todo aquellas relacionadas con las empresas pues así se facilitaría el acceso a estas. Dos décadas más tarde, Griffiths y Guile (2003), señalan la Pedagogía Laboral, como la ciencia normativa, que estudia los problemas que en el mundo del trabajo plantea a la educación y establece normas pedagógicas para su solución. Estos autores, recalcan la necesidad de incluir una materia curricular específica dentro del campo de la Pedagogía, que ofrezca una adecuada formación en contextos empresariales. Los sistemas de calidad que se gestionan en las empresas, la dotan con un peso primordial, de cara a la mejora de sus indicadores. Más aún es crucial gestionar dicha formación de los trabajadores, ya que constituye la base creativa de las estrategias y directrices de las entidades empresariales. En palabras de Guerrero-Cuentas, et ál., (2020), "la importancia que tiene la educación frente al impacto laboral, demanda que el profesional deba mostrar resultados que garanticen el servicio; demostrando lo aprendido en cuanto a los conocimientos con la Universidad que lo formó" (2020, p.31).

Por ello, es obvio por un lado atender a la propia idiosincrasia de esta profesión y, además, otra casuística a tener en cuenta atendiendo a Pineda, et ál., $(2018$, p.313) respecto al mercado de trabajo actual, es que "a pesar de que la economía española se ha ido recuperando, aún presenta cifras altas de desempleo juvenil, por ello, es importante analizar las competencias transversales demandadas y ofertadas en el ámbito laboral, de cara a su inserción efectiva". Por este motivo, la presencia de un profesional de la Pedagogía, adquiere una gran relevancia, para desempeñar puestos destinados a la gestión, impartición y evaluación de procesos formativos de desarrollo profesional, de gestión de competencias y personas, en todos aquellos puestos en los que intervengan procesos de Enseñanza-Aprendizaje (Eraut, 2000). Tales puestos ubicados en un contexto empresarial, son idóneos, dentro de los departamentos de Recursos Humanos en empresas, organismos públicos e instituciones privadas. Ante esta situación, es clara la necesidad de reconceptualizar los ámbitos propios de la Pedagogía en pro, de posibilitar más apertura al mercado de trabajo actual (Álvarez, et ál., 2014), tal y como señalan, González, et ál., "el perfil profesional del pedagogo, ha visto limitado su ejercicio al contexto escolar. La pedagogía y con ella, la figura del pedagogo, deben esforzarse por establecer una distinción clara acerca de sus potencialidades y limitaciones en el ámbito profesional” (2015, p.398).

El presente estudio surge como resultado de una serie de dilemas y problemáticas, recogidas en la revisión de la literatura científica sobre el desconocimiento laboral del profesional de la Pedagogía y, por consiguiente, la apertura al intrusismo profesional, hecho unido a la ausencia de un Colegio Oficial de Pedagogos, en la Comunidad Autónoma de Andalucía. En este sentido, es necesario indagar en las consideraciones que, los futuros profesionales de la Pedagogía tienen sobre la formación recibida y su cercana inserción laboral. Así pues, esto nos permitirá conocer la realidad de sus propias percepciones profesionales, las mismas que servirán como base para establecer medidas de actuación y propuesta de mejora, tanto en el diseño de los planes de estudios y la formación inicial recibida en la Universidad, como una mayor conexión del tejido empresarial con el ámbito más académico.

Para ello, abarcaremos en esta investigación los siguientes objetivos: (1) Conocer las percepciones del alumnado del Grado en Pedagogía de la Universidad de Granada, respecto a funciones y competencias atribuidas a esta titulación, mediante ítems como, "Investiga para la mejora e innovación educativa en la Universidad" y "Es un Gestor de Recursos Humanos en Empresas y Organismos"; (2) Determinar el nivel conocimiento de los alumnos y alumnas en relación al Grado de Pedagogía y su desempeño laboral, a través de ítems como "Se solapa sus funciones con las de otros profesionales" y "Se percibe la definición de esta profesión como ambigua, confusa"; (3) Identificar las expectativas y opiniones de los alumnos sobre los estudios universitarios de Pedagogía, con ítems como "Recomendaría cursar esta titulación universitaria, a un familiar o amigo" y "Le parece una profesión atractiva e interesante"; y (4) Determinar y reconocer la importancia de la imagen social de esta profesión, a través de la concienciación y valoración de su potencial tanto en su perfil como en la amplitud de ámbitos profesionales a desempeñar. 


\section{MÉTODO}

En este epígrafe se van a explicar los diferentes aspectos dentro del método que se han tenido en cuenta a la hora de la realización de la presente investigación. En primer lugar, se encuentra el enfoque metodológico, donde se explica la metodología a seguir y el diseño. A continuación, se exponen las variables tenidas en cuenta en el estudio y se relacionan con las dimensiones. También, se explica la muestra, composición de esta, etc. Por último, se explica el instrumento y cómo se ha realizado el análisis de datos que, más tarde se expondrá.

\section{Enfoque metodológico}

Esta investigación atiende a una metodología descriptiva y cuantitativa, con un diseño no experimental por método de encuesta. Es un estudio que pretende abarcar los conocimientos y percepciones, que versan sobre la Pedagogía y el profesional que la desempeña. Cuestión clave para entender el desconocimiento de la figura profesional del Pedagogo, y poder actuar en consonancia en pro de una mejora de la imagen social que se proyecta a la sociedad. Medida básica para avanzar por el camino adecuado y que permita tomar las decisiones más oportunas. Por ello, el contexto de este estudio se ha focalizado en un centro de formación inicial del profesional de la Pedagogía, en este caso centrado en la Facultad de Ciencias de la Educación de la Universidad de Granada. En los últimos años, la Universidad de Granada ha ido escalando importantes puestos en las clasificaciones y rankings de univerisdades, situándose dentro de las 201-300 mejores universidades del mundo, si se atienden los rankings mundiales, como por ejemplo el Academic Ranking o World Universities, el cual establece anualmente un listado con las 1000 mejores universidades del mundo. Es un indicador imprescindible acceder al conocimiento que el propio alumnado del Grado en Pedagogía, versa sobre sus estudios universitarios y por ende sobre su futuro desempeño profesional.

\section{Variables}

Las variables de esta investigación se correponden con las dimensiones establecidas en el instrumento de recogida de datos para explorar las consideraciones y expectativas que el alumnado de Pedagogía versa en torno al Grado y su desempeño laboral. La estructura del cuestionario "Perfil Profesional del Pedagogo" abarca tres dimensiones: La dimensión Funciones y Competencias -FYC B.1- con 9 ítems (Tabla 2), la dimensión Conocimiento Grado de Pedagogía -GCP B.2- con 9 ítems (Tabla 3), y la dimensión Expectativas y Opiniones - EYO B.3- con 7 ítems (Tabla 4).

Tabla 2: Funciones y competencias (FYC B.1).

\begin{tabular}{|l|l|}
\hline Código & Ítems Dimensión FYC B.1 \\
\hline IMI & Investiga para la mejora e innovación educativa en la Universidad \\
\hline OSC & Es un Orientador Laboral en Organizaciones Socioeducativas y Centros de Empleo \\
\hline ADI & Asesor Didáctico en Instituciones Socioeducativas, diseña, y evalúa materiales \\
\hline ENT & Es un evaluador de Nuevas Tecnologías de Información y Comunicación \\
\hline AIE & Agente de Intervención Educativa a sujetos con Necesidades Educativas Especiales \\
\hline DOE & Se dedica al Diagnóstico, Orientación Escolar y Tutoría \\
\hline DGI & Es Director y Gestor de Instituciones Educativas Formales y No Formales \\
\hline GRH & Es un Gestor de Recursos Humanos en Empresas y Organismos \\
\hline DOC & Es un Docente que imparte Docencia en cualquier Etapa Educativa \\
\hline
\end{tabular}

Tabla 3: Conocimieto grado de Pedagogía (GCP B.2).

\begin{tabular}{|l|l|}
\hline Código & Ítems Dimensión GCP B.2 \\
\hline DLP & Conoce la figura profesional y el desarrollo laboral del Pedagogo \\
\hline PAP & Su perfil académico o profesional, está relacionado con la Pedagogía \\
\hline FPC & Falta de Pedagogos en Centros Escolares \\
\hline FOP & Se solapa sus funciones con otros profesionales \\
\hline PAC & Se percibe la definición de esta profesión como ambigua, confusa \\
\hline EOS & Escasa orientación escolar en Secundaria sobre la elección de titulación universitaria \\
\hline PLE & Contiene esta titulación universitaria, materias de Pedagogía Laboral \\
\hline IOE & Se ofrece orientación, información a alumnos de Pedagogía durante su formación \\
\hline EPO & Pedagogía es escogida en primera opción entre los futuros universitarios \\
\hline
\end{tabular}


Tabla 4: Expectativas y opiniones (EYO B.3). Elaboración propia

\begin{tabular}{|l|l|}
\hline Código & Ítems Dimensión EYO B.3 \\
\hline EPO & Conoce a alguien que esté estudiando o haya estudiado Pedagogía \\
\hline CPT & Conoce algún Pedagogo que esté trabajando en un Centro Escolar o social \\
\hline CPE & Conoce algún Pedagogo que esté trabajando en una empresa u organismo público \\
\hline CEE & Cree que en cada Centro Escolar dispone de al menos un Pedagogo \\
\hline ICE & En un Centro Escolar, ha sido atendido por un Pedagogo al solicitar información \\
\hline RTU & Recomendaría cursar esta titulación universitaria, a familiar o amigo \\
\hline PAI & Le parece una profesión atractiva e interesante \\
\hline
\end{tabular}

\section{Muestra}

La muestra de este estudio de tesis doctoral, está formada por el alumnado que ha cursado estudios de Pedagogía en la Facultad de Ciencias de la Educación de la Universidad de Granada, desde primer curso hasta cuarto de este Grado. En concreto, se ha utilizado "un muestreo finito por conglomerados/estratos artificiales ya preelaborados", de acuerdo con Tallón, Hervás, Polo, Fernández, y Fernández, (2018). En el estudio la muestra participante resultó ser $334(\mathrm{~N}=558)$, es decir, el $60 \%$ de los alumnos matriculados en el Grado de Pedagogía, pertenecientes a esta titulación en el año académico 2019/2020. Estructurado por cursos a través de una muestra mínima a cumplir de participantes por cada grupo, para obtener una correcta distribución. Un $85.90 \%$ del alumnado participante es mujer, y un $14.10 \%$ del alumnado participante es hombre, (Gráfico 1). Con lo cual el grado de Pedagogía, muestra un alto componente femenino a la hora de elegir y cursar estos estudios universitarios. Y situados mayoritariamente en una franja de edad, entre 17-25 años.

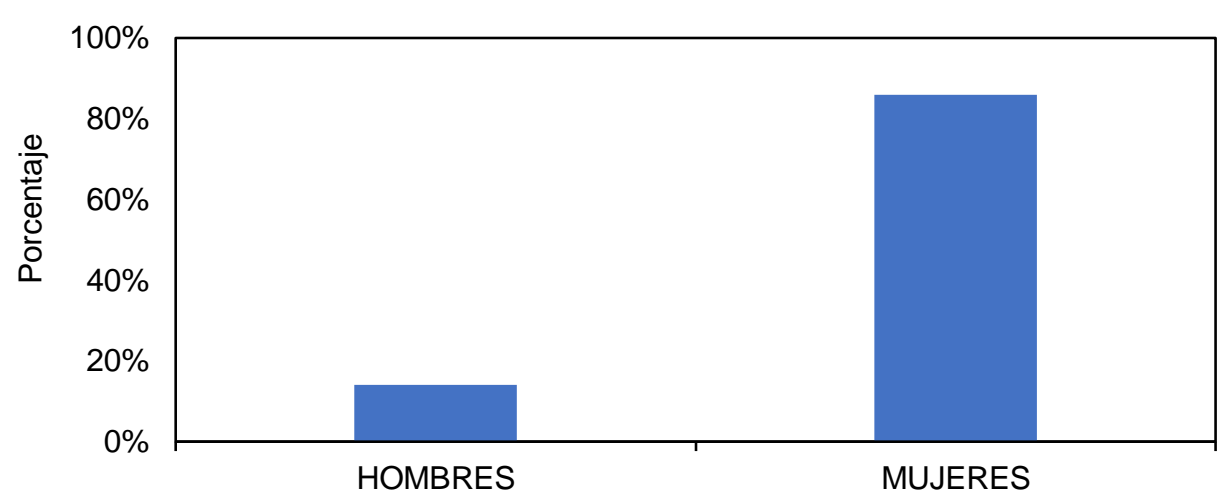

Fig. 1: Distribución de género en la muestra. Elaboración propia

La muestra mínima necesaria para obtener viabilidad a la hora de la realización del estudio se ha superado tanto en los tres primeros cursos, como también en la muestra final total. Por otro lado, teniendo en cuenta el género de los componentes de la muestra, se evidencia de manera clara una descompensación, dado que han sido 287 participantes mujeres, mientras que participantes hombres solo 47 de un total de 334 alumnos encuestados.

\section{Instrumento}

El cuestionario, como instrumento cuantitativo permite la posibilidad de llegar a una muestra más extensa como es nuestro caso, el alumnado de todos los cursos del Grado de Pedagogía. Tiene la ventaja de poder realizar el número de ítems acordes y necesarios para poder extraer toda la información que sea necesaria, y por nuestras propias características de investigación, este instrumento es de elaboración propia, puesto que no hay estudios anteriores o relacionados con nuestra temática, aportando un valor único y diferencial. Su diseño se ha basado, en un test elaborado ad hoc, con preguntas cerradas, tipo escala Likert con cuatro alternativas de respuesta, que van desde muy en desacuerdo a totalmente de acuerdo. Para obtener la validación de contenido ha sido expuesto en reiteradas ocasiones a un juicio de expertos en este ámbito profesional perteneciente a diferentes universidades españolas, hasta alcanzar un alto coeficiente de fiabilidad, indicador de calidad respecto a la elaboración de los ítems de este instrumento. Dicho proceso de elaboración ha conllevado la creación del contenido de cada ítem, de preguntas y de respuestas, formato de la encuesta, dimensiones que agrupan los ítems relacionados, y la coherencia a la hora del diseño de los elementos, siguiendo un orden lógico y secuencial. 


\section{Análisis de datos}

La importancia de la validez y fiabilidad es la certidumbre que proporciona los resultados de la investigación y son cruciales en el proceso de elaboración de cualquier instrumento de investigación (Heale, y Twycross, (2015). Para comprobar dicha eficacia en su construcción, y teniendo en cuenta que a partir de .7, es un buen resultado, hemos utilizado la prueba estadística Alfa de Cronbach, ofreciendo una alta puntuación, en concreto .944 (Gráfico 2). Indicador de una muy alta fiabilidad en la construcción de este instrumento.

Tabla 5: Prueba alfa de cronbach.

\begin{tabular}{|c|c|}
\hline Alfa de Cronbach & N de elementos \\
\hline .944 & 58 \\
\hline
\end{tabular}

En esta investigación, se ha realizado un doble análisis. Por un lado, se encuentran los estadísticos descriptivos y por otro lado, las contingencias obtenidas con la variable Curso. Por ello, se llevó a cabo un minucioso control en la organización de la Pasación a la muestra, para a continuación su correcta clasificación. Nos centraremos en aquellos que ofrecen una mayor significatividad para este estudio, atendiendo al criterio de,

${ }^{* *} \mathrm{P}<.05$ Grado de Significatividad 95\%.

Por último destacamos, el resultado obtenido respecto a las desviaciones típicas de todos los ítems, comprendidas dentro de los límites del intervalo [.789 - 1.465], lo que por su proximidad a la unidad real, garantiza la representatividad estadística de las medias aritméticas asociadas a los mismos.

El tratamiento estadísitico de los datos extraídos se ha realizado mediante el paquete estadístico Statistical Package for the Social Sciences IBM SPSS Statistics 24.0., al ser un recurso idóneo para este trabajo.

\section{RESULTADOS}

Tras la aplicación del Cuestionario, los resultados obtenidos se analizaron en base a dos vertientes, por un lado un estudio de estadísticos descriptivos donde se exponen los porcentajes obtenidos por el alumnado en cada ítem. Dicho estudio evidencia el pensamiento general de los alumnos (Tablas 5,7 y 9). Por otro lado los resultados de las tablas de Contingencia, a través del cruce de los ítems que constituyen el cuestionario, como instrumento cuantitativo de esta investigación, con la variable de corte elegida, "Curso". Es la variable más interesante para comparar resultados, y así conocer las diferencias de opiniones y creencias entre los diferentes cursos de Pedagogía (Tablas 6,8 y 10). A continuación, exponemos los resultados en cada una de las tres dimensiones estudiadas, tanto de los estadísticos descriptivos como de las contingencias obtenidas con la variable Curso. La peimera tabla que se expone es la referente a la Dimensión FYC B.1

Tabla 6: Resultados estadísticos descrptivos dimensión Función y Competencias

\begin{tabular}{|c|c|c|c|c|}
\hline Ítems & $\begin{array}{l}\text { Total de } \\
\text { acuerdo }\end{array}$ & $\begin{array}{l}\text { De } \\
\text { acuerdo }\end{array}$ & $\begin{array}{c}\text { En } \\
\text { desacuerdo }\end{array}$ & $\begin{array}{l}\text { Total en } \\
\text { desacuerdo }\end{array}$ \\
\hline Investiga para la mejora e innovación educativa en la Universidad & $30.80 \%$ & $46.70 \%$ & $17.40 \%$ & $3.30 \%$ \\
\hline $\begin{array}{l}\text { Es un orientador laboral en organizaciones socioeducativas y } \\
\text { centros de empleo }\end{array}$ & $37.10 \%$ & $40.40 \%$ & $15.90 \%$ & $6.30 \%$ \\
\hline $\begin{array}{l}\text { Es un asesor didáctico en instituciones socioeducativas, diseña y } \\
\text { evalúa materiales y recursos didácticos }\end{array}$ & $43.40 \%$ & $34.70 \%$ & $15.00 \%$ & $6.90 \%$ \\
\hline $\begin{array}{l}\text { Es un evaluador de nuevas } \\
\text { tecnologías de información y comunicación }\end{array}$ & $18.30 \%$ & $41.90 \%$ & $31.70 \%$ & $7.80 \%$ \\
\hline $\begin{array}{l}\text { Es un agente de intervención educativa a sujetos con } \\
\text { necesidades educativas especiales }\end{array}$ & $45.50 \%$ & $32.90 \%$ & $13.50 \%$ & $8.10 \%$ \\
\hline Se dedica al diagnóstico, orientación escolar y tutoría & $45.80 \%$ & $33.80 \%$ & $10.50 \%$ & $9.90 \%$ \\
\hline $\begin{array}{l}\text { Es un director y gestor de instituciones educativas formales y no } \\
\text { formales }\end{array}$ & $27.50 \%$ & $40.70 \%$ & $25.10 \%$ & $6.60 \%$ \\
\hline Es un gestor de recursos humanos en empresas y organismos & $21.60 \%$ & $44.30 \%$ & $26.30 \%$ & $7.80 \%$ \\
\hline Es un docente que imparte docencia en cualquier etapa educativa & $21.30 \%$ & $29.90 \%$ & $31.70 \%$ & $17.10 \%$ \\
\hline
\end{tabular}


Observamos que el perfil del Pedagogo, en base a las valoraciones de los alumnos del Grado de Pedagogía, predominan tres funciones. La primera, indica que el profesional de la Pedagogía, está muy vinculado a la orientación escolar como función prioritaria. Respecto a la segunda función y competencias, resaltan que el Pedagogo realiza actuaciones para detectar e intervenir en alumnos que presentan dificultades de diversa índole en los centros escolares. Y la tercera más elegida predomina la función de asesor didáctico en instituciones, en las cuales diseña y evalúa materiales, así como proporcionar recursos acordes para mejorar los procesos de enseñanza-aprendizaje. Los ítems con mayor significatividad $\left(^{* *}\right)$ en esta dimensión son: Investiga para la mejora e innovación educativa en la Universidad; Es un agente de intervención educativa a sujetos con necesidades educativas especiales y Es un docente que imparte docencia en cualquier etapa educativa. A continuación, se muestran los resultados obtenidos referentes a la Dimensión CGP. B2

Tabla 7: Contingencia variable Curso y dimensión Funciones y Competencias. Elaborado a partir de SPSS 24.0

\begin{tabular}{|c|c|c|c|c|}
\hline \multirow[t]{2}{*}{ Ítems } & \multicolumn{4}{|c|}{ Curso (CUR) } \\
\hline & g.I. & $x^{2}$ & $p$ (sig.) & Casos válidos \\
\hline IMI & 9 & $19.763^{\mathrm{a}}$ & $.019^{\star \star}$ & 328 \\
\hline OSC & 9 & $13.240^{\mathrm{a}}$ & .152 & 333 \\
\hline ADI & 9 & $13.215^{\mathrm{a}}$ & .153 & 334 \\
\hline ENT & 9 & $16.018^{\mathrm{a}}$ & .067 & 333 \\
\hline AIE & 9 & $21.324^{\mathrm{a}}$ & $.011^{\star \star}$ & 334 \\
\hline $\mathrm{DOE}$ & 9 & $14.983^{\mathrm{a}}$ & .091 & 334 \\
\hline DGI & 9 & $10.638^{\mathrm{a}}$ & .301 & 334 \\
\hline GRH & 9 & $13.737^{\mathrm{a}}$ & .132 & 334 \\
\hline $\mathrm{DOC}$ & 9 & $25.826^{\underline{a}}$ & $.002^{* \star}$ & 334 \\
\hline
\end{tabular}

Tabla 8: Estadísticos descriptivos dimensión Conocimiento Grado de Pedagogía.

\begin{tabular}{|l|c|c|c|c|}
\hline \multicolumn{1}{|c|}{ Ítems } & $\begin{array}{c}\text { Total de } \\
\text { acuerdo }\end{array}$ & $\begin{array}{c}\text { De } \\
\text { acuerdo }\end{array}$ & $\begin{array}{c}\text { En } \\
\text { desacuerdo }\end{array}$ & $\begin{array}{c}\text { Total en } \\
\text { desacuerdo }\end{array}$ \\
\hline Conoce la figura profesional del Pedagogo & $21.60 \%$ & $44.00 \%$ & $29.30 \%$ & $5.10 \%$ \\
\hline Su perfil está relacionado con la Pedagogía & $36.20 \%$ & $40.10 \%$ & $15.30 \%$ & $8.10 \%$ \\
\hline Falta de Pedagogos en los centros escolares & $57.10 \%$ & $20.68 \%$ & $13.79 \%$ & $8.09 \%$ \\
\hline Se solapan funciones con otros profesionales & $56.24 \%$ & $23.08 \%$ & $12.29 \%$ & $8.39 \%$ \\
\hline Se percibe esta profesión ambigua y confusa & $57.50 \%$ & $24.50 \%$ & $10.50 \%$ & $7.50 \%$ \\
\hline $\begin{array}{l}\text { Escasa orientación respecto a la elección de titulación-salidas } \\
\text { laborales }\end{array}$ & $57.14 \%$ & $24.88 \%$ & $12.29 \%$ & $5.69 \%$ \\
\hline $\begin{array}{l}\text { Contiene materias relacionadas con Pedagogía Laboral- } \\
\text { Empresarial }\end{array}$ & $16.50 \%$ & $41.60 \%$ & $32.00 \%$ & $9.90 \%$ \\
\hline $\begin{array}{l}\text { Se ofrece orientación a estudiantes de Pedagogía durante su } \\
\text { formación }\end{array}$ & $15.00 \%$ & $35.90 \%$ & $39.50 \%$ & $9.60 \%$ \\
\hline $\begin{array}{l}\text { Cree que la titulación de Pedagogía es escogida como primera } \\
\text { opción }\end{array}$ & $7.49 \%$ & $19.18 \%$ & $48.75 \%$ & $24.58 \%$ \\
\hline
\end{tabular}

En esta tabla se refleja, de las cuestiones planteadas respecto al conocimiento que se tiene del propio Grado de Pedagogía a los alumnos encuestados, que existen cuatro ítems que reciben una puntuación muy alta cerca del $60 \%$, en la opción más positiva de todas, totalmente de acuerdo/excelente. Todas estas puntuaciones, que superan la mayoría de las respuestas, son indicativas que lo más llamativo o lo que más se conoce del Grado de Pedagogía, son acepciones negativas. Se reconoce que es una profesión que genera duda y confusión, a la hora de definir lo qué es y su quehacer profesional, (57.50\%). Y además señala tres factores muy importantes respecto a su desempeño profesional, como son la escasa presencia de pedagogos ejerciendo en secundaria, (57.14\%) y por consiguiente no han sido asesorados en dicha etapa educativa respecto a la elección de grado universitario y salidas profesionales, $(57.14 \%)$. Labor primordial de la función del Pedagogo, que tal y como hemos indicado en la tabla anterior, es la más elegida por los estudiantes del Grado de Pedagogía participantes en este estudio.

Por último, también se hace patente la existencia de un importante intrusismo profesional dentro de la Pedagogía, (56.24\%) por la multitud de funciones similares con otras titulaciones, que cuenta con un mayor reconocimiento social. Una cuestión a tener en cuenta, es la coincidencia en puntuación, de dos ítems, como son la falta de pedagogos en centros escolares y la escasa orientación escolar en secundaria, ambos con un $57.14 \%$. Este dato indica que el uno depende del otro, manifestando una acción-reacción. Otra característica de importante reflexión, puesto que ha sido la más elegida en la opción de respuesta negativa, en desacuerdo/regular con un $48.75 \%$ es, cree que la titulación de Pedagogía es escogida como primera opción, 
entre los futuros universitarios. De nuevo una valoración negativa, puesto que han respondido que no es un Grado de primera opción, casi la mitad de los participantes.

Tabla 9: Contingencias variable curso y dimensión conocimiento grado de Pedagogía.

\begin{tabular}{|c|c|c|c|c|}
\hline \multirow{2}{*}{ Ítems } & \multicolumn{4}{|c|}{ Curso (CUR) } \\
\cline { 2 - 5 } & g.I. & $\mathrm{x}^{2}$ & $\mathrm{p}(\mathrm{sig})$. & Casos válidos \\
\hline DLP & 9 & $18.283^{\underline{a}}$ & $.032^{* *}$ & 334 \\
\hline PAP & 9 & $26.289^{\underline{a}}$ & $.002^{* *}$ & 333 \\
\hline FPC & 9 & $26.714^{\underline{a}}$ & $.002^{* *}$ & 333 \\
\hline FOP & 9 & $20.786^{\underline{a}}$ & $.014^{* *}$ & 334 \\
\hline PAC & 9 & $18.841^{\underline{a}}$ & $.027^{* *}$ & 334 \\
\hline EOS & 9 & $23.319^{\underline{a}}$ & $.006^{* *}$ & 334 \\
\hline PLE & 9 & $20.125^{\underline{a}}$ & $.017^{* *}$ & 334 \\
\hline IOE & 9 & $38.404^{\underline{a}}$ & $.000^{* *}$ & 334 \\
\hline EPO & 9 & $21.722^{\underline{a}}$ & $.010^{* *}$ & 334 \\
\hline
\end{tabular}

Lo más característico es que todos los ítems, son muy significativos $\left({ }^{* *}\right)$, con lo cual la importancia de su significado y relevancia para la investigación, queda patente. Con respecto a la Dimensión EYO. B3 es importante destacar que, a pesar del desconocimiento de esta titulación, un $41.90 \%$ recomendaría cursar este Grado. Los ítems con mayor significatividad $\left({ }^{* *}\right)$ en esta dimensión son tres: Conoce algún Pedagogo que esté trabajando en una empresa u organismos públicos; En el caso de aconsejar a un familiar o amigo, le recomendaría cursar esta titulación universitaria y Le parece una profesión atractiva e interesante.

Tabla 10: Estadísticos descriptivos dimensión Expectativas y Opiniones.

\begin{tabular}{|l|c|c|c|c|}
\hline \multicolumn{1}{|c|}{ Ítems } & $\begin{array}{c}\text { Total de } \\
\text { acuerdo }\end{array}$ & $\begin{array}{c}\text { De } \\
\text { acuerdo }\end{array}$ & $\begin{array}{c}\text { En } \\
\text { desacuerdo }\end{array}$ & $\begin{array}{c}\text { Total en } \\
\text { desacuerdo }\end{array}$ \\
\hline $\begin{array}{l}\text { Conoce a alguien que esté estudiando o haya } \\
\text { estudiado Pedagogía }\end{array}$ & $58.40 \%$ & $12.30 \%$ & $12.30 \%$ & $17.10 \%$ \\
\hline $\begin{array}{l}\text { Conoce algún Pedagogo que esté trabajando en un } \\
\text { centro escolar o entidades sociales }\end{array}$ & $33.50 \%$ & $19.80 \%$ & $22.80 \%$ & $24.00 \%$ \\
\hline $\begin{array}{l}\text { Conoce algún Pedagogo que esté trabajando en una } \\
\text { empresa u organismos públicos }\end{array}$ & $21.00 \%$ & $13.20 \%$ & $26.30 \%$ & $39.50 \%$ \\
\hline $\begin{array}{l}\text { Cree que en cada centro escolar o entidad educativa, } \\
\text { dispone al menos de un Pedagogo }\end{array}$ & $12.30 \%$ & $25.40 \%$ & $38.90 \%$ & $22.80 \%$ \\
\hline $\begin{array}{l}\text { Cuando ha necesitado información de un centro escolar } \\
\text { ha sido atendido por un Pedagogo }\end{array}$ & $10.20 \%$ & $22.20 \%$ & $31.40 \%$ & $36.20 \%$ \\
\hline $\begin{array}{l}\text { En el caso de aconsejar a un familiar o amigo, le } \\
\text { recomendaría cursar esta titulación universitaria }\end{array}$ & $30.80 \%$ & $41.90 \%$ & $19.20 \%$ & $8.10 \%$ \\
\hline Le parece una profesión atractiva e interesante & $50.30 \%$ & $29.00 \%$ & $11.40 \%$ & $9.30 \%$ \\
\hline
\end{tabular}

Tabla 11. Contingencia viable Curso y dimensión Expectativas y Opiniones.

\begin{tabular}{|c|c|c|c|c|}
\hline \multirow{2}{*}{ Ítems } & \multicolumn{4}{|c|}{ Curso (CUR) } \\
\cline { 2 - 5 } & g.l. & $\mathrm{x}^{2}$ & $\mathrm{p}$ (sig.) & $\begin{array}{c}\text { casos } \\
\text { válidos }\end{array}$ \\
\hline EPO & 9 & $7.264^{\mathrm{a}}$ & .610 & 334 \\
\hline CPT & 9 & $6.606^{\mathrm{a}}$ & .678 & 334 \\
\hline CPE & 9 & $18.455^{\underline{a}}$ & $.030^{* *}$ & 334 \\
\hline CEE & 9 & $14.257^{\underline{a}}$ & .113 & 332 \\
\hline ICE & 9 & $16.577^{\underline{a}}$ & .056 & 334 \\
\hline RTU & 9 & $30.049^{\underline{a}}$ & $.000^{* *}$ & 334 \\
\hline PAI & 9 & $21.824^{\underline{a}}$ & $.009^{* *}$ & 334 \\
\hline
\end{tabular}




\section{DISCUSIÓN}

Tras la exposición de los resultados, podemos discernir que los datos obtenidos atienden a los objetivos marcados para este estudio. En primer lugar, se ha profundizado en las creencias, valoraciones y pensamientos de los estudiantes del Grado de Pedagogía, sobre su propia profesión. Valoración muy importante ya que, esos ideales o acepciones en torno a la figura del Pedagogo y su desarrollo profesional, que el alumnado tiene, será proyectada por los mismos al resto de la sociedad, y, en consecuencia, la sociedad tendrá dicho conocimiento en torno a esta titulación. Por ello, es vital seguir investigando en esta temática, dada la escasa literatura existente, con la finalidad de realizar una proyección hacia la sociedad, hacia el mercado laboral, clarificante y eficaz. Dar a conocer esta información al resto de la comunidad educativa y empresarial, es muy importante para ayudar a esclarecer y reconocer cuál es la figura profesional del Graduado en Pedagogía dentro de la sociedad. Esto permitirá ayudar en la toma de decisiones de futuros empleadores, sobre la inclusión del perfil del Pedagogo y su potencialidad laboral (Tallón, et ál., 2017).

Según se desprende de los datos aportados y en base a los objetivos del estudio, se puede concluir que la titulación de Pedagogía, desde el contexto universitario de Granada, se percibe por parte del estudiantado que la cursa, como una profesión centrada en tres funciones básicas: la orientación escolar, la intervención con necesidades especiales de diversa índole y la asesoría didáctica. Esto es, enmarcada predominante en la educación formal de forma generalizada, como uno de sus principales ámbitos de desempeño profesional. De igual modo, los participantes de esta investigación consideran que la docencia no es una labor específica del desarrollo profesional del Pedagogo; pero sí es un gestor en centros educativos de programas de orientación escolar y académica así también como vocacional; y es responsable de las labores de tutoría y su coordinación con los tutores, dentro de los Departamentos de Orientación.

A medida que se asciende de un curso a otro, las puntuaciones varían mostrando un mayor conocimiento de esta figura en otros ámbitos, más allá del escolar, como en empresas u otros organismos. En este sentido sería necesario, una formación más completa y global para toda la titulación, desde el primer curso hasta el último. Como, por ejemplo, complementar la formación inicial de esta titulación, con la introducción en el currículo actual, de materias como Pedagogía Laboral, y Formación en las Organizaciones, impartidas actualmente en otras Universidades, posibilitando un itinerario profesional más para el Pedagogo, el sector empresarial. Casi la mayoría del alumnado no elige estos estudios universitarios como primera opción a la hora de decidir la titulación para su futuro profesional. A pesar de no ser una titulación escogida en primera opción, los alumnos y las alumnas que la están cursando consideran atractiva e interesante su labor y, es más, la recomendarían. Esto ofrece la visión, de ser un Grado desconocido inicialmente, ya que los datos demuestran que una vez que han mantenido algún tipo de información o contacto, están muy satisfechos con ella (Tallón, 2017). En este sentido, autores como Collin, et ál., (2013) y Corney y Reid, (2007) manifiestan que la formación inicial del profesorado podría mejorar su grado de prácticas experimentales con la finalidad de lograr una mejor aplicación de las metodologías activas, para que así los retos y necesidades que la sociedad actual demanda puedan solventarse de una manera más eficaz. Es crucial el ajuste entre formación inicial del profesional del ámbito educativo en sus respectivas universidades y puestos de trabajos ofertados por la sociedad y, por ende, el mercado laboral. En este contexto, Erstad, et ál., enfatizan que "la labor del profesor universitario no se limita a transmitir información, sino en potenciar la actividad del estudiante para filtrar la información, organizarla, procesarla, construir los contenidos de aprendizaje y desarrollar habilidades y competencias" (2021, p.40).

El amplio abanico de funciones y ámbitos de actuación profesional, puede constituir en sí, la razón de la ambigüedad, desconocimiento y/o confusión que gira en torno a la definición de qué es la Pedagogía y cuál es su misión. En comparación con otras profesiones, esta titulación tiene un carácter multidisciplinar, a través de tres grandes ámbitos de intervención, (ámbito formal, el ámbito no-formal y el ámbito empresarial), y en cada uno, desarrollan diversas funciones y puestos (Tallón, et ál., 2018). En este sentido, los futuros pedagogos aseguran no tener un conocimiento suficiente de la figura profesional y desarrollo laboral de esta profesión, reconociendo la ambigüedad que provoca. Junto con la escasez de puestos laborales y falta de visibilidad del Pedagogo en activo, así como un acusado intrusismo con profesiones afines. Este pensamiento generalizado incide en la imagen profesional del Graduado en Pedagogía de forma negativa, puesto que el propio estudiantado así lo expresa, con lo cual crea confusión en cuanto a la formación inicial universitaria y la necesidad de una mayor conexión con el amplio elenco de salidas laborales, resultando una profesión poco reconocida y valorada. Tampoco mejora dicha situación el hecho tan sólo de existir, tres Colegios Oficiales de Pedagogía en el territorio español, el Colegio Oficial de Pedagogos y Psicopedagogos de Valencia, el Colegio Oficial de Pedagogos de Islas Baleares y el Colegio de Pedagogos de Cataluña, que defienda y vele por los derechos de los profesionales de la Pedagogía. Estar amparados por un organismo que proteja su imagen profesional, constituiría un aspecto muy positivo en pro de su labor. 


\section{CONCLUSIONES}

Este tipo de estudios son fundamentales para impulsar el reconocimiento de esta profesión, concienciar en la promoción de actuaciones que mejoren esta condición, e impulsar la contribución de la Pedagogía en los diferentes ámbitos en los cuales puede ejercerse profesionalmente, sin establecer ambigüedades, confusión o confrontaciones en su acción laboral. Ha sido vital analizar las percepciones de los propios estudiantes de este Grado, ofreciendo la imagen que tienen sobre sí mismos y su futuro desarrollo profesional. Se convierte en un indicador de calidad que promoverá la mejora, puesto que posibilita incidir en la imagen que actualmente se proyecta de esta titulación. Siendo fundamental extender este tipo de estudios a otros contextos universitarios, donde se muestren más evidencias al respecto; lo que obligue a reflexionar a toda la comunidad educativa para investigar en la mejora formativa de nuevos planes de estudios, que aborden y recojan los diferentes ámbitos de inserción laboral de la Pedagogía, más allá de "la Orientación Escolar" y promuevan un mayor conocimiento y vinculación con el tejido empresarial (Prácticum, experiencias de colaboración, Spin off, etc.), como un motor de arranque que conciencie a la sociedad sobre la labor de esta profesión, y se sumen a nuevas investigaciones al respecto, creando un bagaje propio que pueda impulsar desde diversas entidades públicas y privadas una visión positiva y eficiente del desarrollo profesional que ofrece la Pedagogía a la comunidad científica y educativa.

\section{AGRADECIMIENTOS}

Este artículo está derivado de la tesis doctoral titulada: "Análisis del perfil del pedagogo y su inserción laboral en la provincia de Granada, desde la perspectiva de los ámbitos de actuación profesional". Universidad de Granada. (https:// handle.net/10481/47663 acceso el 30 de marzo de 2021), por lo que los autores agradecen a la Universidad de Granada y resto de instituciones implicadas la colaboración, interés y apoyo en todo el desarrollo de la presente investigación.

\section{REFERENCIAS}

Alvarez, J. G., Losada, A. S., y Comesaña, J. M. C., Prospects for Social Employment Insertion of Graduates in Pedagogy in the Autonomous Community of Galicia. From the University to the Labour Market, Procedia-Social and Behavioral Sciences, 139, 412-418 (2014)

Boletín Oficial del Estado, Orden del 22 de abril de 1983 por la que se aprueba la implantación del plan de estudios de la Sección de Pedagogía de la Facultad de Filosofía y Letras de la Universidad de Granada, Madrid, España: Gaceta de Madrid (1983)

Boletín Oficial Junta de Andalucía, Decreto 158/1992 del 1 de septiembre, por el que se crea la Facultad de Ciencias de la Educación en la Universidad de Granada, Sevilla, España: Consejería de Educación y Ciencia de Andalucía (1992)

Collin, S., Karsenti, T., y Komis, V., Reflective practice in initial teacher training: Critiques and perspectives, Reflective practice, 14(1), 104-117 (2013)

Corney, G., y Reid, A., Student teachers' learning about subject matter and pedagogy in education for sustainable development, Environmental Education Research, 13(1), 33-54 (2007)

Eraut, M., Non-formal learning and tacit knowledge in professional work, British Journal of Educational Psychology, 70(1), 113-136 (2000)

Ermenc, K. S., Vujisić, N. Ž., y Spasenović, V., Theory, practice and competences in the study of pedagogy-views of Ljubljana and Belgrade university teachers, Center for Educational Policy Studies Journal, 5(2), 35-55 (2015)

Erstad, O., Miño, R., y Rivera-Vargas, P., Educational practices to transform and connect schools and communities. Prácticas educativas para transformar y conectar escuelas y comunidades, https://doi.org/10.3916/C66-202101, Comunicar, 66, 9-20 (2021)

Facultad Ciencias de la Educación, Granada, Evaluación de las Titulaciones: Licenciado en Pedagogía, Granada: Universidad de Granada (2003)

Griffiths, T., y Guile, D., A connective model of learning: the implications of work process knowledge, European Journal of Educational Research, 1(1), 156-173 (2003)

González, C., Martínez, P., y González, N., El perfil formativo del Graduado en Pedagogía: La visión del Alumnado. Profesorado, Revista de Curriculum y Formación del Profesorado, 19(1), 394-412 (2015)

Guerrero-Cuentas, H.R., Morales-Ortega, Y., Nuñez-Rios, G.P., y Medina-Fonseca, E.D., Impacto de la resignificación de la práctica pedagógica investigativa y del currículo de graduados de pedagogía de instituciones de educación superior en Barranquilla-Colombia, https://dx.doi.org/10.4067/S0718-50062020000200029, Formación Universitaria, 13(2), 29-38 (2020)

Heale, R., y Twycross, A., Validity and reliability in quantitative studies, http://dx.doi.org/10.1136/eb-2015102129, Evidence-Based Nursing, 18(3), 66-67 (2015) 
López, M.I., Rubio, S., y Díaz, E., Cambios producidos por la adaptación al EEES en la carga de trabajo de estudiantes de Psicología, https://doi.org/10.22550/REP77-1-2019-02, Revista Española de Pedagogía, 77(272), 163-176 (2019)

Medina, J. C., y González, J. A., Graduation Profiles of Pedagogy Programmes According to the Current Educational Policies, https://doi.org/10.5539/ies.v12n6p83, International Education Studies, 12(6), 83-93 (2019)

Miller, J., y Baker, D., Career orientation and pedagogical training: conservatoire undergraduates' insights, https://doi.org/10.1017/S0265051706007194, British Journal of Music Education, 24(1), 5 (2007)

Nassif, R., Pedagogía General, Madrid: Editorial Cincel (1981)

Petrie, P., Extending 'pedagogy', Journal of Education for Teaching, 31(4), 293-296 (2005)

Pineda, P., Ciraso, A., y Armijos, M., Competencias para la empleabilidad de los titulados en Pedagogía, Psicología y Psicopedagogía: un estudio comparativo entre empleadores y titulados, https://doi.org/10.22550/REP76-2-2018-06, Revista Española de Pedagogía, 76 (270), 313-333 (2018)

Robinson, T. E., y Hope, W. C., Teaching in Higher Education: Is There a Need for Training in Pedagogy in Graduate Degree Programs?, Research in Higher Education Journal, 21, 1-11 (2013)

Saldarriaga, O., Historia de la pedagogía como historia de la cultura: ¿entre la historia de las ideas y la historia social?, Anuario Colombiano de Historia Social y de la Cultura, 44.1 101-123 (2013)

Santos, M., y Álvarez, J., La inserción laboral de los pedagogos en el marco de las políticas activas de empleo, https://doi.org/10.1590/0104-4060.49146, Educar em Revista, Curitiba Brasil, Edición especial (1), 115-131 (2017)

Tallón, S., Análisis del Perfil del Pedagogo y su Inserción Laboral en la provincia de Granada desde la perspectiva de los Ámbitos de Actuación Profesional, Tesis Doctoral, Universidad de Granada (2017)

Tallón, S., Cáceres, M., y Raso, F., Análisis del perfil profesional del Pedagogo en el ámbito Empresarial Español, mediante la técnica de Análisis de Puestos de Trabajo, Revista Estudios Hemisféricos y Polares, 8(2), 1-20 (2017)

Tallón, S., Hervás, M., y otros tres autores, El perfil Formativo y Profesional del Graduado en Pedagogía. Perspectiva actual de su Inserción Laboral, https://doi.org/10.17060/ijodaep.2018.n1.v2.1230, International Journal of Developmental and Educational Psychology, Monográfico, 1, 295-306 (2018)

Tolipov, U. Q., Main quality levels of pedagog professional competence, Central Asian Journal of Education, 3(1), 2-18 (2019)

Valladares, L., Transdisciplinariedad y pedagogía: apuntes para el debate sobre la (in)disciplina del pedagogo, https://doi.org/10.15359/rep.15-1.4, Revista Ensayos Pedagógicos, 15(1), 81-102 (2020)

Zhuina, D. V., Diagnostics of career orientation peculiar for the personality of pedagogy students, Life Science Journal, 11(8), 586-589 (2014) 